\title{
Washington y Ginebra llegan a Buenos Aires: notas sobre la historia del hábito de fumar y su medicalización
}

\author{
Washington and Geneva arrive in Buenos Aires: notes on the \\ history of the habit of smoking and its medicalization
}

\section{Diego Armus}

Profesor de Historia latinoamericana/Department of History/Swarthmore College. College Avenue, 500 - Swarthmore PA 19081 - USA

darmus1@swarthmore.edu
ARMUS, Diego. Washington y Ginebra llegan a Buenos Aires: notas sobre la historia del hábito de fumar y su medicalización. História, Ciências, Saúde - Manguinhos, Rio de Janeiro, v.22, n.1, jan.-mar. 2015, p.293-302.

\section{Resumen}

Durante gran parte del siglo XX tanto los gobiernos civiles como los militares no encontraron en el tabaquismo un tema prioritario. Recién en la última década del siglo el movimiento internacional contra el cigarrillo, liderado por la Organización Mundial de la Salud, organizaciones norteamericanas y académicos, empezó a tener algún impacto en la escena política argentina. Fue en ese contexto que un nuevo grupo profesional logró impulsar la constitución de un amplio bloque político antitabaco. En ese proceso, el voluntarismo centrado en programas individuales para dejar de fumar que había marcado gran parte de las iniciativas del siglo XX, terminó desplazado por políticas públicas destinadas a producir ambientes libres de humo y a combatir la exposición pasiva al humo de tabaco ajeno.

Palabras clave: historia; consumo de cigarrillos; políticas públicas; Argentina.

\begin{abstract}
For much of the twentieth century both the civilian and military governments did not consider smoking a priority concern. It was only in the last decade of the twentieth century that the international movement against cigarettes, led by the World Health Organization, US organizations and academics, began to have some impact on Argentina's political scene. It was in this context that a new professional group managed to foment the creation of a broad anti-smoking political bloc. In this process, voluntarism focused on individual programs to quit smoking that had marked much of the initiatives of the twentieth century, ended up being replaced by public policies designed to ensure smoke free environments and combat passive smoking.
\end{abstract}

Keywords: history; smoking; public policies; Argentina. 
$\mathrm{E}$ 29 de septiembre de 2005 y el 13 de diciembre de 2010 la Legislatura de la Ciudad Autónoma de Buenos Aires sancionó las leyes 1.799 y 3.718 con el objetivo de regular el consumo, comercialización y publicidad del tabaco. Ambas leyes enmarcaban sus detalles en un explícito discurso que jerarquizaba la prevención y asistencia de la salud pública y la salud individual de los habitantes de la ciudad.

Su articulado es exhaustivo y vale la pena presentarlo en conjunto toda vez que se trata de instrumentos legales que revelan una notable atención al detalle. Con estas leyes se prohíbe todo tipo de patrocinio o financiación de actividades culturales, deportivas o educativas, con libre acceso de empresas o personas, cuya actividad principal sea la fabricación, distribución o promoción de productos derivados del tabaco. Se prohíbe, sin excepción, el expendio, provisión y/o venta de productos elaborados con tabaco a los menores de 18 años, sea para consumo propio o para su comercialización. Se prohíbe la venta de productos elaborados con tabaco en establecimientos educativos de enseñanza primaria y secundaria y centros de salud. Se prohíbe la comercialización y distribución de productos de uso o consumo corriente entre niños/as y adolescentes que por su denominación, formato o envase, constituyan una evidente o subliminal inducción a generar o difundir el hábito de fumar. Se prohíbe fumar en todos los espacios cerrados con acceso público, entre los que se listan restaurantes, bares y confiterías, lugares en que se brindan servicios de computadoras y/o conexión a internet, con o sin anexos de cafetería, salas de recreación, shoppings y paseos de compras cerrados, salas de teatro y cine, centros culturales, salas de fiestas o de uso público, en general en las que se permita la entrada a menores de 18 años, recintos con cabinas telefónicas, cajeros automáticos y otros espacios de uso público de reducido tamaño, estaciones terminales y/o de transbordo de micro ómnibus de mediana y larga distancia, vehículos de servicio público de transporte colectivo de pasajeros, estaciones de trenes subterráneos, instituciones deportivas y gimnasios. Se establecen sanciones a los titulares o responsables de establecimientos comerciales que vendan tabaco a personas menores de edad. Se prohíbe la comercialización y publicidad del tabaco en cualquiera de sus modalidades en las áreas públicas de la ciudad. Se prohíben los anuncios publicitarios de productos elaborados con tabaco, ya sea para su venta, promoción y entrega en forma gratuita. Se exceptúa de esta última prohibición a establecimientos que comercialicen cigarrillos, quedando estos comercios obligados a incluir en los envases de esos productos mensajes, fácilmente legibles y prominentes, preparados por las autoridades sanitarias, destinados a instruir al consumidor sobre las nocivas consecuencias del humo de tabaco en la salud.

Las leyes también establecen la realización de campañas de información y esclarecimiento en establecimientos educacionales y medios de comunicación masiva acerca de los riesgos que implica el consumo del tabaco así como el impulso y la planificación de procedimientos de control para asegurar el cumplimiento de las normas de publicidad, comercialización, distribución y consumo de cigarrillos y productos similares. Explícitamente, las leyes aspiran a desarrollar una conciencia social sobre el derecho de los no fumadores a respirar aire sin la contaminación ambiental producida por el humo del tabaco en los espacios cerrados, a impulsar la formulación de programas de asistencia gratuita para las personas interesadas en dejar de fumar, a difundir el conocimiento de las patologías vinculadas con el tabaquismo, sus consecuencias y las formas de prevención y tratamiento. Incluso articulan una expresa crítica 
a las estrategias de la industria tabacalera que promueven el hábito de fumar recurriendo a campañas publicitarias que las leyes califican como desleales, engañosas e ilícitas.

El exhaustivo contenido de estas leyes condensa muy claramente el quiebre de una tradición casi secular en que el hábito de fumar fue celebrado de modo sostenido y practicado con intensidad. Esa suerte de subcultura estuvo muy bien alimentada no solo por los intereses de la industria tabacalera y su crecientemente sofisticada publicidad en diarios, revistas, radio y televisión sino también por el tango, la literatura urbana y el cine nacional y extranjero. En la perdurable presencia de esta subcultura también contaron, y de modo decisivo, los propios fumadores. Sus experiencias y sensibilidades con el hábito de fumar produjeron un sinnúmero de narrativas personales que apuntaban a dar cierta racionalidad a la decisión y elección de fumar. Las más recurrentes se articulaban en torno del control del stress, la relajación, el placer, el autogobierno de los deseos y el cuerpo, la responsabilidad individual, la masculinidad, la independencia femenina, la reafirmación juvenil.

Algunos ejemplos ilustran bien esta historia de continuidades a todo lo largo del siglo XX. En 1900 las propagandas de variadas marcas de cigarrillos abundaban en diarios y revistas, revelando que se trataba de un objeto de consumo masivo, tal vez de los primeros que entrenaba al consumidor en el moderno ejercicio cotidiano de elegir entre productos similares. En 1922 se estrenó "Fumando espero", uno de los veinte tangos más celebrados del siglo XX donde el acto de fumar realizado por una mujer, apasionadamente enamorada, se presenta como "un placer genial y sensual". En la literatura urbana de las décadas del 1940 al 1960, de Jorge Luis Borges a Julio Cortázar, el cigarrillo marca infinidad de escenas de la vida en Buenos Aires. Y a mediados de la década del 1990, el autor de la guía turística The Lonely Planet escribía que en "el Parque de Palermo hay quienes hacen jogging con un cigarrillo en la boca". Expandir esta galería de situaciones es una tarea relativamente sencilla puesto que el hábito de fumar saturó prácticamente todas las dimensiones del diario vivir a lo largo del siglo. Su perdurable omnipresencia solo puede sorprender a los que ahora tienen menos de 20 o 25 años.

En 2014 estas imágenes parecen extemporáneas. Al despuntar el siglo XXI, en los años en que se sancionaron las leyes 1.799 y 3.718, lo que fue el celebrado hábito de fumar terminó signado por la sospecha, la enfermedad y la muerte. Así, el hombre confiado, seguro y autosuficiente, la mujer liberada o el joven empeñado en obtener el reconocimiento adulto algunas de las tantas asociaciones que saturan los años cuando fumar era, en gran medida, una fiesta - han sido reemplazados por hombres y mujeres que fuman y por esa razón son percibidos por la sociedad como individuos débiles, irracionales, presas de una incontrolable adicción que atenta contra la salud pública.

De ser un hábito aceptado y percibido como inocuo fumar cigarrillos pasó a ser una adicción dañina, medicalizada y criminalizada. Estas notas, de tono exploratorio, proponen acercarse a la dinámica de este cambio, centrando su atención en los actores que permitieron alterar en apenas unos pocos años una historia de continuidades que recorre casi todo el siglo XX. ${ }^{1}$

Dos asuntos fueron decisivos en la consolidación del nuevo escenario que recién empezó a cuajar a fines del siglo XX y que permitió que la legislatura de la Ciudad Autónoma de Buenos Aires aprobara las leyes 1.799 y 3.718, ambas bien expresivas del emergente y global consenso antitabaco. 
De una parte, se trata de la consolidación de la figura del fumador pasivo, esto es, el que no fuma pero que debe convivir con el humo producido por otros que sí fuman. A comienzos de los años 1990, la figura del fumador pasivo permitió articular y justificar tímidas iniciativas estatales regulatorias que no lograron plasmarse en políticas e instrumentos legales, concretos, pero que prepararían el terreno para que en la década siguiente, ya en el siglo XXI, fructificaran en políticas concretas. El segundo asunto remite al nuevo escenario creado por las acciones impulsadas por la Organización Mundial de la Salud - un actor supranacional que en 2003 impulsó y logró la aprobación del Convenio Marco para el Control del Tabaco. Ambos - la figura del fumador pasivo y la globalización de la campaña antitabaco - contribuyeron decisivamente en la consolidación de una moralidad secular que comenzará a valorar, con una intensidad probablemente desconocida en el siglo XX, salud y fitness, conciencia de riesgo, cambio de conductas y un notable reforzamiento de la idea de no hacer daño a otros con el acto de fumar (Katz, 1997, p.298; Rozin, 1997, p.380).

En Buenos Aires - y esto en modo alguno es una excepcionalidad de la modernidad argentina - no faltan antecedentes que desde comienzos del siglo XX advierten sobre los intentos de regular el consumo de cigarrillos. Fueron iniciativas totalmente marginales e ineficaces. Hacia los años 1930 y 1940 hubo intentos en los círculos académicos, también fracasados, por consensuar la asociación del cigarrillo y el cáncer y por transformar el tabaquismo en una cuestión de salud pública en torno de la cual debían articularse campañas específicas y masivas. Entre 1950 y 1960, el tema fue ganando presencia entre algunos sectores de la comunidad científica mundial, fundamentalmente a partir de reportes producidos en los Estados Unidos y Gran Bretaña sobre los efectos del cigarrillo en la salud (Brandt, 2007, p.211-239; Hilton, 2000, p.179-202). Su impacto en Argentina, tanto en los medios científicos como en la sociedad, fue insignificante. Con todo, para esos años se empezaba a vislumbrar la emergencia de un cambio de perspectivas y en ese nuevo contexto lo que solía pensarse como un hábito terminó siendo etiquetado como dependencia psicológica y, más tarde, como adicción.

Fue recién hacia el final del siglo XX, cuando en los círculos científicos y en la sociedad y la cultura de Buenos Aires avanzó con paso firme la idea de que fumar era dañino para la salud y que aún los no-fumadores también sufrían los efectos nocivos del humo producido por los fumadores. Entrado el siglo XXI la nocividad del cigarrillo ya se había galvanizado como sentido común, un escenario impensable solo dos décadas atrás.

Inevitablemente este nuevo y crecientemente globalizado consenso tomaría perfiles propios en cada realidad nacional. En general, las variables que moldearon las peculiaridades de cada una de esas realidades respondieron a contextos y coyunturas políticas específicas y locales. Allí contaron las tradiciones legales; las características del estado de bienestar y de los sistemas de atención de la salud - de los armados en torno al Estado como único proveedor a los que se consolidaron de la mano de mecanismos de mercado; las tradiciones burocráticas - de las muy eficientes a las ineficientes, de las muy fuertes a las muy débiles; por último, las creencias y prácticas en relación a cuestiones de privacidad y autonomía de los individuos (Feldman, Bayer, 2004, p.2-7).

Cuatro cuestiones devinieron claves en esas políticas: ¿Cuánto control debía imponerse en la publicidad de cigarrillos? ¿Cuán intensas debían ser las restricciones a fumar en los 
espacios públicos? ¿Qué políticas impositivas debían aplicarse a la venta de cigarrillos? ¿Qué iniciativas y estrategias debían impulsarse para ayudar a los fumadores a dejar de fumar de modo individual?

Durante gran parte del siglo XX ninguna de las iniciativas antitabaco originadas en el Estado o en la sociedad civil logró contrabalancear las eficaces acciones de los tres actores claves que por distintos motivos alentaban y alimentaban el hábito de fumar en Buenos Aires: (1) las compañías tabacaleras; (2) las provincias productoras de tabaco; (3) las agencias del Estado federal interesadas en los impuestos recaudados por la venta de cigarrillos. A estos tres actores deben sumarse los deseos, percepciones y necesidades de los propios fumadores que armaban, gestionaban, manejaban y justificaban su relación con el cigarrillo en términos de hábito, adicción, placer, incluso daño a la salud personal.

La ineficacia de estas iniciativas encuadra bien con el lugar económico y social que el cigarrillo ha tenido en el Buenos Aires moderno. Algunos datos lo ilustran con elocuencia.

La manufactura local de los cigarrillos describe una curiosa trayectoria: empezó a fines del siglo XIX como un sector de negocios definitivamente doméstico, organizado en torno de varias fábricas pequeñas y medianas que producían cerca de 100 marcas de cigarrillos de tabaco negro. Entre 1920 y 1930 se instalaron en Buenos Aires compañías inglesas y norteamericanas. Con ellas se fueron haciendo un lugar, cada vez más prominente, los cigarrillos de tabaco rubio y de mezcla. En ese nuevo contexto, y por un par de décadas, las empresas tabacaleras nacionales compitieron con las extranjeras. Hacia el último tercio del siglo XX y en consonancia con una tendencia mundial, el sector manufacturero del cigarrillo se fue concentrando hasta terminar manejado por dos subsidiarias de las poderosísimas corporaciones tabacaleras: British American Tobacco y Phillip Morris International.

Desde fines del siglo XIX Buenos Aires registra comparativamente altos niveles de consumo de cigarrillos per cápita, probablemente más altos que los del mundo angloamericano donde la pipa, los cigarros y el tabaco de mascar fueron populares y donde el consumo de cigarrillos se consolidará recién después de la Primer Guerra Mundial. También las mujeres porteñas parecen haber sido fumadoras tempranas cuando se las compara con las anglosajonas que recién empezaron a hacerlo de modo significativo a partir de la década de 1930. A todo lo largo del siglo XX, el consumo de cigarrillos per cápita no hará más que consolidarse y aumentar. Así, y como una elocuente evidencia del prominente lugar sociocultural que el hábito de fumar tenía en la vida de los habitantes de Buenos Aires, en 2007 un estudio advertía sobre la elevada proporción de médicos que seguían siendo fumadores aun cuando en esos años ya era difícil ignorar las campañas antitabaco impulsadas por algunas agencias del Estado y organizaciones de la sociedad civil (Zilbersztejn et al., 2007, p.113-114).

Por último, cabe tomar nota que la Argentina ha estado produciendo tabaco. En 2004, el $2 \%$ de la producción mundial de hojas de tabaco se originó en seis provincias del noroeste y noreste argentinos. Allí la agricultura del tabaco ya se había configurado como un sector económico destacado que permitía la subsistencia a un cuarto de millón de personas trabajando en unidades productivas familiares pequeñas y medianas. Desde 1972, esa agricultura de subsistencia ha estado subsidiada con un impuesto al consumo de cigarrillos consistente en el 7\% del precio de venta de cada paquete de cigarrillos. $80 \%$ de lo recaudado se destinaba a los agricultores tabacaleros y el 20\% restante a iniciativas orientadas a la diversificación 
agrícola. Con esta política de subsidios, el Estado facilitó un mecanismo que logró mantener bajos los precios de los cigarrillos mientras facilitaba la reproducción de un sistema productivo basado en muy modestas unidades económicas familiares (Mejía, Pérez-Stable, 2006, p.50).

La ineficacia casi secular de las iniciativas antitabáquicas empezó a modificarse en las últimas dos décadas del siglo XX y los primeros años del XXI cuando se crearon nuevas organizaciones sociales activamente enfocadas en la lucha contra del cigarrillo, avanzaron las restricciones legales sobre la publicidad y el consumo de cigarrillos en lugares públicos, aumentaron los estudios epidemiológicos que buscaban dar legitimidad a las campañas antitabaco y se firmaron convenios internacionales contra el consumo de tabaco. A diferencia de otros lugares, en particular los Estados Unidos, los juicios de ex-fumadores a las compañías tabacaleras no fueron parte de esas inéditas y por primera vez eficaces iniciativas reguladoras del consumo de cigarrillos. Frente a ese nuevo escenario, la industria no se quedó quieta y desplegó una infinidad de respuestas no solo en el terreno del marketing sino también en el de la justificación discursiva de la práctica de fumar como un derecho individual ejercido con plena conciencia. Promovió estrategias como "La cortesía de elegir" o "Convivencia en armonía", ambas diseñadas con el objetivo de no solo obstaculizar la aprobación de legislación que pusiera fin a la exposición pasiva al humo de tabaco ajeno en restaurantes y bares sino también mantener la aceptación social del consumo de tabaco (Sebrié et al., 2005, p.5). En esa línea, y desde fines de los años 1990, el lobby tabacalero alentó programas de prevención del uso de tabaco entre los jóvenes. Se trataba de un enfoque que pivoteaba sobre la voluntad personal del fumador joven o la de quienes regenteaban los lugares de venta de cigarrillos que no debían venderlos a menores de 18 años. Con ese énfasis apuntaban a liberar a la industria de cualquier responsabilidad por sus sofisticadas campañas de publicidad y comercialización dirigidas a potenciales fumadores jóvenes. Con ese énfasis, también, subrayaban que el derecho a fumar era una legítima opción y un derecho individual que cada fumador podía gestionar a su gusto.

Desde fines del siglo XIX hay preocupaciones sobre el cigarrillo y sus efectos en la salud. Se trató, sin duda, de preocupaciones intermitentes. Esto ha sido así en Argentina y en muchos otros países del mundo. La Alemania Nazi es, probablemente, un momento bastante excepcional en la historia del hábito de fumar en Occidente durante el siglo XX toda vez que fueron años marcados por una muy activa y explicitada campaña antitabaco (Proctor, 2011, p.159-170). En Buenos Aires, esas preocupaciones recorren todo el siglo pero siempre fueron muy modestas y marginales, por lo general llevadas a cabo por médicos y académicos. Su agenda era informativa y educativa. El mensaje dominante centraba en señalar que fumar hacía mal a la salud y que el modo de dejar el hábito, vicio o adicción - los términos cambian con el paso del tiempo - era motivar y dar suficientes recursos racionales, emocionales y de apoyo profesional que permitieran al fumador hacer uso de su voluntad y embarcarse en la aventura individual de dejar de fumar. No eran iniciativas que apuntaban a incidir aunque más no fuera tangencialmente en la producción de políticas públicas.

Algunos ejemplos de estas iniciativas, originadas en la sociedad civil o en el Estado, ilustran bien esta historia de continuidades. En la década de 1930, el médico e investigador Angel Roffo - uno de los pioneros a nivel mundial en la asociación entre cigarrillos y cáncer impulsó campañas antitabaco desde su Instituto de Medicina Experimental utilizando 
recursos financieros estales y estrategias de información y educación del público en general mediante diarios, revistas y programas de radio. Años más tarde, en 1963, la iglesia adventista organizó su primer curso para dejar de fumar en cinco días. A mediados de los años 1980, el Rotary Club de Argentina conformó un comité especial contra el consumo de cigarrillos. En 1986, la Liga Argentina contra el Cáncer creó el Chau Pucho Club luego de haber lanzado una ingeniosa, original y agresiva campaña de difusión en los medios invitando a los fumadores a sumarse a sus grupos de apoyo para dejar de fumar. En 1987, se fundó la Unión Antitabáquica Argentina, integrada por un grupo de médicos y profesionales de la salud - algunos trabajando en agencias del Estado - que muy tímidamente comenzaría a sumar a la divulgación de estrategias personales para dejar de fumar otras centradas en el lobby a legisladores y la promoción de políticas públicas antitabaco. Para esos años, estos grupos de profesionales participaban en la recién creada Coalición Latinoamericana de Control del Tabaco, una alianza regional apoyada por la American Cancer Society.

Fue en la última década del siglo XX que comenzó a tomar forma un nuevo consenso antitabaco que sin duda compagina muy mal con las fracasadas iniciativas alentadas por profesionales y organizaciones de la sociedad civil. Eran los inicios de una nueva etapa en la historia de los empeños contra el consumo de tabaco. Una nueva generación de profesionales comenzaría a abogar por políticas antitabaco con estrategias renovadas. Se trataba de expertos y profesionales, estrechamente vinculados con organizaciones internacionales y extranjeras, que habían hecho estadías de entrenamiento en instituciones académicas y/o organizaciones enfocadas en cuestiones de salud pública en los Estados Unidos o en Ginebra y que sabrían aprovechar las crecientes y en gran medida recientes oportunidades para obtener apoyos financieros destinados a solventar campañas de abogacía de políticas públicas contra el cigarrillo. En 2000, la Fundación Interamericana del Corazón lideró un proceso de renovación de la existente Coalición Latinoamericana de Control del Tabaco, poniendo a disposición fondos y expertos y, lo más importante, impulsando una agenda que unos años más tarde apuntaría a ratificar e implementar los lineamientos aprobados por el Convenio Marco para el Control del Tabaco de la Organización Mundial de la Salud. En 2006, la Iniciativa para Reducir el Uso del Tabaco, impulsada por la fundación norteamericana Bloomberg Philanthropies, comenzaría a apoyar financieramente a organizaciones no-gubernamentales, a alentar y monitorear el cumplimiento de iniciativas legales orientadas al establecimiento de espacios libre de humo e investigar las dimensiones económicas del negocio del tabaco y la evaluación y control de las actividades de la industria tabacalera. En este nuevo contexto, en 2007, se formó la Alianza Libre de Humo-Argentina, una coalición integrada por cerca de 100 organizaciones. En 2009, solo dos años mas tarde, la coalición había triplicado sus miembros y logrado involucrarse activamente en el programa nacional de control de tabaco dependiente del Ministerio de Salud (Marcet Champagne et al., 2010, p.336).

$\mathrm{Al}$ igual que con las iniciativas de la sociedad civil, las originadas en las agencias del Estado fueron marginales e inefectivas durante gran parte del siglo XX y solo en los últimos años registraron un cambio de estrategia y agenda. Entre 1966 y 2003, bajo gobiernos civiles y dictaduras militares, hubo cerca de veinte iniciativas parlamentarias destinadas a regular el consumo de cigarrillos pero todas ellas fueron eficientemente bloqueadas por el 
lobby tabacalero, por las presiones de las provincias productoras de tabaco y por las agencias nacionales interesadas en recaudar impuestos.

En 1966 fue introducido en el Congreso Nacional argentino el primer proyecto de ley para la regulación del tabaco. El proyecto, que no logró ser aprobado, requería la colocación obligatoria de una etiqueta de advertencia sanitaria en todos los paquetes de cigarrillos. En 1970, el gobierno promulgó la ley 18.604, que ponía fin a la publicidad de los cigarrillos en la radio, la televisión y los cines y establecía multas para los infractores. Sin embargo, esta ley estuvo en vigencia solo durante un año. En 1973 y 1974 dos proyectos de ley retomaban la iniciativa de 1966 y requerían no solo la colocación de una etiqueta de advertencia sanitaria en los envases de los productos de tabaco sino también en toda la publicidad que anunciara cigarrillos. Estos proyectos no fueron aprobados debido a la intervención de la Cámara de la Industria del Tabaco. En 1977, y tal como ocurrió en los Estados Unidos, la Cámara de la Industria del Tabaco creó voluntariamente un Código de Autorregulación Publicitaria. Se trataba de un débil e inefectivo listado de condiciones, destinado a evitar la aprobación de legislación que realmente permitiera restringir la publicidad del tabaco. En 1976 y 1979 el Ministerio de Bienestar Social de la Nación preparó dos proyectos de ley orientados a regular el contenido de la publicidad del tabaco. En respuesta a esas iniciativas representantes de la industria del tabaco presionaron sobre funcionarios del gobierno aseverando que el voluntariamente establecido Código de Autorregulación Publicitaria, preparado por la industria tabacalera, era suficiente. Finalmente, ambos proyectos fueron archivados. En 1986 el Congreso Nacional aprobó la ley 23.344, que reafirmaba el inefectivo y voluntario Código de Autorregulación Publicitaria de la industria del tabaco y requería la colocación en los paquetes de cigarrillos de la débil etiqueta de advertencia sanitaria que decía "Fumar es perjudicial para la Salud".

En 1992, la Octava Conferencia Mundial sobre Tabaco o Salud se llevó a cabo en la ciudad de Buenos Aires. Activistas locales por el control del consumo de tabaco intentaron aprovechar la coyuntura para presionar la aprobación en el Congreso Nacional de un proyecto de ley, muy amplio, que en 1990 había sido presentado por el diputado Aldo Neri. El 30 de septiembre de 1992 la Ley Neri fue aprobada en el Congreso Nacional. Sin embargo, el lobby tabacalero contraatacó, movilizando el concurso de la Sociedad Interamericana para la Libertad de Expresión Comercial y la Asociación Argentina de Agencias de Publicidad, de algunos consultores científicos y de legisladores de las provincias tabacaleras. El argumento utilizado enfatizó que la relación de causalidad entre tabaco y enfermedad no estaba probada. Días más tarde, el 10 de octubre, y revelando el poder que en la década de 1990 todavía tenía el frente de fuerzas defensoras del derecho a fumar, la ley fue vetada por el presidente Carlos Menem.

Lo que ocurrió en Argentina en los últimos años no ha sido nada excepcional. Con diferencias nacionales, y en algunos países de modo más efectivo y rápido que en otros, el nuevo consenso fogoneado desde Washington y Ginebra y alentado localmente por más y más políticos y organizaciones no gubernamentales ha logrado modificar significativamente el secular hábito de fumar. En 2003, Argentina firmó el Convenio Marco para el Control del Tabaco de la Organización Mundial de la Salud. Su ratificación, sin embargo, fue resistida en el Congreso Nacional, donde los votos de las provincias tabacaleras contaban de modo decisivo en las alianzas del partido de gobierno. Así, el Poder Ejecutivo optó por no priorizar 
el tema. Hacia 2006 había una sola ley nacional que enfatizaba en algunas restricciones sobre la publicidad de cigarrillos en ciertos lugares. En la Ciudad Autónoma de Buenos Aires la legislatura local aprobó las leyes a las que se hizo referencia al comienzo de estas notas - en 2005 y 2010 - que colocaban a la ciudad junto a las provincias que más convincentemente impulsaban agresivas y sofisticadas políticas antitabaco, sustancialmente distintas a las todavía tímidas iniciativas a nivel nacional.

En la última década el gobierno federal articuló dos discursos al mismo tiempo. De una parte, no presionó a los representantes de las provincias tabacaleras a que apoyen una ley realmente comprehensiva para el control del consumo del tabaco. De otra, el Ministerio de Salud trató, sin éxito, de impulsar una ley diseñada siguiendo los estándares mínimos definidos en 2003 por el Convenio Marco para el Control del Tabaco de la Organización Mundial de la Salud. Fue recién en 2012 cuando la Argentina ratificó el convenio y el Congreso Nacional aprobó una nueva ley nacional, aun más explícita y detallada que la aprobada por la Legislatura de la Ciudad Autónoma de Buenos Aires en 2010. Desde su aprobación, los resultados de este conjunto de iniciativas legislativas han sido notables. Si bien Argentina continúa siendo uno de los países con más alta prevalencia de tabaquismo de América Latina, entre 2006 y 2012 y a nivel mundial, fue uno de los países donde se registró una de las más acentuadas reducciones en el consumo de cigarrillos per cápita (Ng, 2014, p.183-192).

El caso argentino descubre una historia de continuidad con un cambio brusco localizado temporalmente en los últimos diez años. Durante gran parte del siglo XX, la regulación del consumo de cigarrillos no logró hacerse un lugar en la política formal. Tanto los gobiernos civiles - de signo conservador, radical, peronista o desarrollista - como las dictaduras militares las moderadas o las brutalmente autoritarias - no encontraron en el tabaquismo un tema prioritario. Recién en la última década del siglo, y liderado por la Organización Mundial de la Salud, organizaciones norteamericanas como Bloomberg Philanthropies y la American Cancer Society y militantes de grupos académicos, el movimiento global contra el cigarrillo empezó a tener algún impacto en la escena política argentina. Fue en ese nuevo contexto internacional que un nuevo grupo profesional de abogacía logró impulsar la constitución de un bloque político antitabaco donde participaron legisladores de prácticamente todas las fuerzas políticas representadas en el Congreso Nacional. Así, y con el telón de fondo de un consenso internacional muy proclive a la medicalización y criminalización del hábito de fumar, la política local - tanto la parlamentaria como la impulsada por el Poder Ejecutivo se alineó sin dudar detrás de agresivas y comprehensivas políticas contra el consumo de cigarrillos. En ese proceso el voluntarismo antitabaco, centrado en programas individuales para dejar de fumar que había marcado gran parte de las iniciativas del siglo XX, terminó desplazado por políticas públicas destinadas a producir ambientes libres de humo y combatir la exposición pasiva al humo de tabaco ajeno.

\section{NOTA}

\footnotetext{
${ }^{1}$ Este artículo adelanta algunos de los temas de un libro en preparación, tentativamente titulado Fumar en Buenos Aires: una historia en el siglo XX, donde me propongo discutir discursos, políticas y experiencias asociadas con la práctica de fumar.
} 


\section{REFERENCIAS}

BRANDT, Alan.

The cigarette century: the rise, fall, and deadly persistence of the product that defined America. New York: Basic Books. 2007.

FELDMAN, Eric; BAYER, Ronald.

Liberal states, public health, and the tobacco question. In: Feldman, Eric et al. Unfiltered: conflicts over tobacco policy and public health. Cambridge, Massachusetts: Harvard University Press. p.1-7. 2004.

HILTON, Matthew.

Smoking in British popular culture: 1800-2000.

Manchester: Manchester University Press. 2000.

KATZ, Solomon.

Secular morality. In: Brandt, Allan; Rozin, Paul (Ed.). Morality and health. New York: Routledge. p.297-330. 1997.

MARCET CHAMPAGNE, Beatriz et al. The role of organized civil society in tobacco control in Latin America and the Caribbean. Salud Pública de México, v.52, supl.2, p.330-339. 2010.

MEJÍA, Raúl; PÉREZ-STABLE, Eliseo.

Tobacco epidemic in Argentina: the cutting edge of Latin America. Prevention and Control, v.2, n.1, p.49-55. 2006.
NG, Marie et al.

Smoking prevalence and cigarette consumption in 187 countries, 1980-2012. Journal of the American Medical Association, v.322, n.2, p.183192. 2014.

PROCTOR, Robert.

Golden holocaust: origins of the cigarette catastrophe and the case for abolition. Berkeley: University of California Press. 2011.

ROZIN, Paul.

Moralization. In: Brandt, Allan; Rozin, Paul. Morality and health. New York: Routledge. p.379-401. 1997.

SEBRIÉ, Ernesto et al.

Tobacco industry dominating national tobacco policy making in Argentina, 1966-2005. San Francisco: Center for Tobacco Control Research and Education/University of California. Disponible en: http//repositories.cdlib.org/ctcre/tcpmi/ Argentina2005. Acceso en: ene. 2005. 2005.

ZILBERSZTEJN, Horacio et al.

Tabaquismo en médicos de la República Argentina: Estudio TAMARA. Revista Argentina de Cardiología, v.75, n.2, p.109-116. 2007. 


\section{ERR A T A}

A pedido do autor, no texto "Washington y Ginebra llegan a Buenos Aires: notas sobre la historia del hábito de fumar y su medicalización", de Diego Armus, publicado em História, Ciências, Saúde - Manguinhos, v.22, n.1, jan.-mar. 2015:

- na página 301, segundo parágrafo, sexta linha, onde se lê "Fue recién en 2012 cuando la Argentina ratificó el convenio y el Congreso Nacional aprobó una nueva ley nacional", leia-se "Fue recién en 2012, y sin haber ratificado el convenio, cuando el Congreso Nacional aprobó una nueva ley nacional".

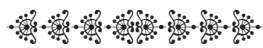

